\title{
Gastrectomia vertical (Sleeve) em paciente pós-transplante hepático
}

\section{Vertical gastrectomy (Sleeve) in a patient after hepatic transplantation}

Mayara Magry Andrade da Silva1 ${ }^{1}$ Carlos Eduardo Lopes Soares ${ }^{1}$. João Odilo Gonçalves Pinto ${ }^{2}$. Leonardo Adolpho de Sá Sales ${ }^{2}$. Fernando Antônio Siqueira Pinheiro². José Huygens Parente Garcia².

1 Universidade Federal do Ceará (UFC), Fortaleza, Ceará, Brasil. 2 Hospital Universitário Walter Cantídio (HUWC/UFC/ EBSERH), Fortaleza, Ceará, Brasil.

\section{RESUMO}

Objetivos: Descrever o primeiro caso de cirurgia bariátrica (Gastrectomia Vertical - Sleeve) em paciente submetido a transplante hepático, para tratamento de obesidade grau III e esteatose severa do enxerto no Hospital Universitário Walter Cantídio (HUWC). Metodologia: Após a aprovação do Comitê de Ética e Pesquisa do HUWC, foram coletados no prontuário os seguintes dados pré e pós-operatórios: peso, altura, índice de massa corpórea (IMC), ultrassonografia (USG) de abdome, transaminases, perfil lipídico e metabólico. Resultados: Paciente, feminino, 62 anos, pós-operatório de transplante hepático, foi submetida a gastrectomia vertical (Sleeve) em junho de 2019 para tratamento de obesidade grau III (IMC 40,1). Biópsia hepática pré-operatória evidenciou esteatose hepática macrovesicular grave com USG compatível com esteatose severa e exames de função hepática discretamente elevados. Evoluiu no pós-operatório sem intercorrências, recebendo alta no $2^{\circ}$ pós-operatório (PO). Durante seguimento ambulatorial houve perda de $24 \mathrm{~kg}$ em 5 meses e USG de abdome de controle evidenciou esteatose hepática leve. Provas de função hepática, perfil lipídio e metabólico encontravam-se dento dos padrões da normalidade. Conclusões: A obesidade é uma causa importante de disfunção de enxerto ortotópico de fígado. A cirurgia bariátrica configura como terapêutica segura e eficaz no tratamento da obesidade e esteatose hepática grave.

Palavras-chave: Obesidade. Transplante de fígado. Fígado gorduroso.

\section{ABSTRACT}

Objectives: To describe the first case of bariatric surgery (Vertical Gastrectomy - Sleeve) in a patient undergoing liver transplantation, for the treatment of grade III obesity and severe graft steatosis at the Hospital Universitário Walter Cantídio (HUWC). Methodology: After approval by the HUWC Ethics Committee, the following pre and postoperative data were collected from the chart: weight, height, body mass index (BMI), ultrasound (USG) of the abdomen, transaminases, lipid and metabolic profile. Results: Female, 62 years old, after liver transplant surgery, underwent vertical gastrectomy (Sleeve) in June 2019 for treatment of grade III obesity (BMI 40.1). Preoperative liver biopsy showed severe macrovesicular hepatic steatosis with USG compatible with severe steatosis and slightly elevated liver function tests. He evolved in the postoperative period without complications, being discharged in the 2nd PO. During outpatient follow-up, there was a loss of $24 \mathrm{~kg}$ in 5 months and USG control abdomen showed mild hepatic steatosis. Evidence of liver function, lipid and metabolic profile were within the normal range. Conclusions: Obesity is an important cause of orthotopic liver graft dysfunction. Bariatric surgery is a safe and effective therapy for the treatment of obesity and severe hepatic steatosis.

Keywords: Obesity. Liver transplantation. Fatty liver.

Autor correspondente: Mayara Magry Andrade da Silva, Avenida da Abolição, 2950, Meireles, Fortaleza, Ceará. CEP: 60165-078. Telefone: +55 85 98141-1222. E-mail: mayaramasilva@hotmail.com

Conflito de interesses: Não há qualquer conflito de interesses por parte de qualquer um dos autores.

Recebido em: 20 Set 2020; Revisado em: 13 Abr 2021; Aceito em: 29 Jun 2021. 


\section{INTRODUÇÃO}

O transplante hepático em humanos foi descrito pela primeira vez em 1963 por T. E. Starzl e atualmente é o procedimento de escolha para o tratamento de pacientes com doença hepática crônica ou aguda, quando a conduta conservadora não é efetiva. A sobrevida global em 3 anos pode chegar a $80 \%{ }^{1}$

Dentre as indicações em adultos, podemos citar alguns grupos principais: doenças hepáticas colestáticas crônicas, doenças hepatocelulares crônicas, doenças hepáticas metabólicas, doenças hepáticas vasculares, tumor primário do fígado e trauma. ${ }^{1,2}$ Apesar da principal causa de cirrose hepática com indicação de transplantes estar associada a infecção por vírus $\mathrm{B}$ e C, atualmente a esteato hepatite não alcoólica (NASH) já é a segunda causa principal de transplante nos Estados Unidos (EUA). ${ }^{1,3,4}$

A incidência de obesidade tem sido cada vez maior nos países ocidentais $^{5}$ associado ao aumento na prevalência de doença hepática gordurosa não alcoólica (DHGNA), ${ }^{6,7}$ fortemente associada a síndrome metabólica e corresponde a um espectro de alterações hepáticas que compreendem: esteatose, NASH, fibrose ou cirrose. ${ }^{6}$

Os principais fatores de risco para desenvolvimento de DHGNA incluem principalmente: obesidade, diabetes melittus tipo II e dislipidemia. Outros fatores como drogas (amiodarona, corticosteroides, estrógenos sintéticos, tamoxifeno, nifedipina), toxinas ambientais (produtos químicos), cirurgias (by-pass jejuno-ileal, derivação bilio-pancreática; ressecção extensa do intestino delgado) e o vírus $\mathrm{C}$ da hepatite também estão associados a esta condição. ${ }^{6,7}$

Um terço dos pacientes com NASH em estágio inicial progredirá para fibrose dentro de 5 a 10 anos após o diagnóstico clínico, fato este que justifica sua associação com a maioria dos transplantes de fígado não virais. ${ }^{8,9}$

Após o transplante, as taxas de recorrência da obesidade são frequentes, justificando a necessidade de identificação dos grupos de risco e a otimização de seu tratamento, assim como tratamento de comorbidades metabólicas associadas. Este cuidado deve ser realizado antes e após o transplante hepático para manutenção de um enxerto saudável a longo prazo. ${ }^{5}$

Este trabalho foi aprovado pelo Comitê de Ética e Pesquisa do Hospital Universitário Walter Cantídio (HUWC), protocolo $\mathrm{n}^{\circ}$ 041.02.2020, e tem como objetivo relatar a experiência inicial no tratamento de esteatose hepática em fígado transplantado com a utilização da cirurgia bariátrica com técnica de gastrectectomia vertical laparoscópica (Sleeve) no Hospital Universitário Walter Cantídio- CE.

\section{RELATO DE CASO}

Paciente do sexo feminino, 62 anos, IMC 40,1, com histórico de obesidade desde a infância, foi submetida a transplante hepático em novembro de 2015 por cirrose criptogênica.
O transplante foi realizado pela técnica de piggyback com doador cadáver e ocorreu com boa evolução pós-operatória, sem complicações clínicas ou cirúrgicas. Durante seguimento ambulatorial, foi observado aumento importante de peso chegando a 113,5 kg em 5 meses (IMC 40,1) associado a resistência à insulina, dislipidemia e aumento das enzimas hepáticas configurando presença de esteatose hepática em enxerto.

Foram realizadas medidas dietéticas e acompanhamento nutricional sem sucesso com redução mínima do peso $(4 \mathrm{~kg}$ em 7 meses). Em decorrência das comorbidades, insucesso nas medidas clínicas para controle do peso e risco elevado de comprometimento do enxerto por esteatose hepática, foi optado por indicar intervenção cirúrgica bariátrica como tratamento de escolha.

A paciente foi submetida a gastrectomia vertical videolaparoscópica (Sleeve) em junho de 2019 (43 meses após o transplante). Evoluiu sem intercorrências no pós-operatório com início da dieta e retorno dos imunossupressores no $2^{\circ}$ dia de pós operatório (DPO), com boa adaptação alimentar. Recebeu alta hospitalar no $2^{\circ} \mathrm{DPO}$ e manteve seguimento ambulatorial.

Durante seguimento, houve perda de $24 \mathrm{~kg}$ em 5 meses e USG de abdome de controle evidenciou esteatose hepática leve. Provas de função hepática, perfil lipídio e metabólico encontravam-se dento dos padrões da normalidade.

\section{Técnica cirúrgica}

O procedimento cirúrgico foi realizado sob anestesia geral com intubação orotraqueal, a paciente foi mantida em decúbito dorsal horizontal com o cirurgião posicionado entre os membros inferiores da paciente.

A cirurgia teve inicio com a confecção de pneumoperitôneo e punção de trocateres abdominais (1 trocater de $15 \mathrm{~mm}, 1$ trocater de $12 \mathrm{~mm}$ e 3 trocaters de $5 \mathrm{~mm}$ ) seguida por lise de grande quantidade de aderências entre o lobo esquerdo do fígado e a parede anterior do estômago (Figura 1).

Figura 1. Lise de aderências entre lobo hepático esquerdo e parede anterior do estômago com pinça ultrassônica.

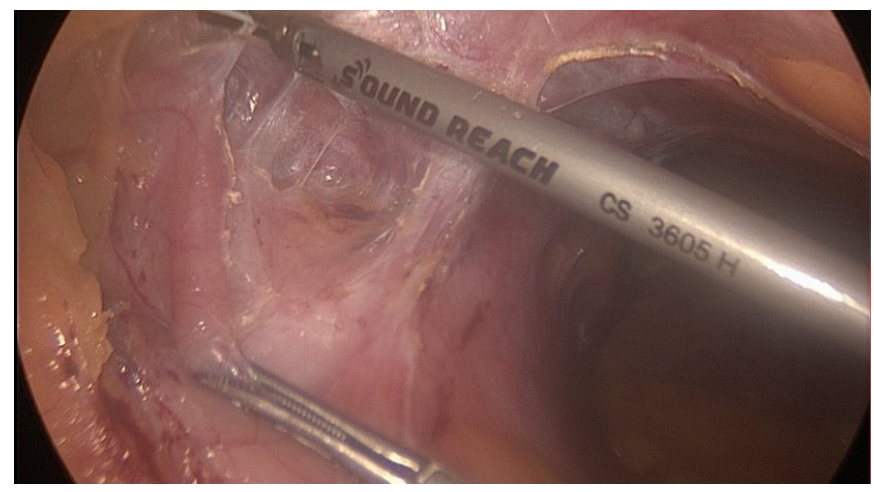


Foi seccionado o grande omento justo a grande curvatura do estômago do antro até o ângulo de His utilizando a pinça ultrassônica (Figura 2). A confecção do tubo gástrico foi realizada com grampeador laparoscópico e calibrada com sonda de Fouchet número 32. O grampeamento foi iniciado com carga verde de $60 \mathrm{~mm}$ a $4 \mathrm{~cm}$ do piloro, seguido por carga verde no segundo disparo e azul nos 4 disparos subsequentes (Figura 3). Foi realizada a revisão cuidadosa na hemostasia, biópsia hepática e optado por não deixar dreno abdominal. A peça cirúrgica foi retirada pelo trocater abdominal de $15 \mathrm{~mm}$ e realizada síntese apenas de pele.

O tempo cirúrgico foi de 110 min e o tempo anestésico de 130 min. Não houve intercorrências ou necessidade de transfusão.

Figura 2. Grande curvatura após secção de grande omento com pinça ultrassônica.

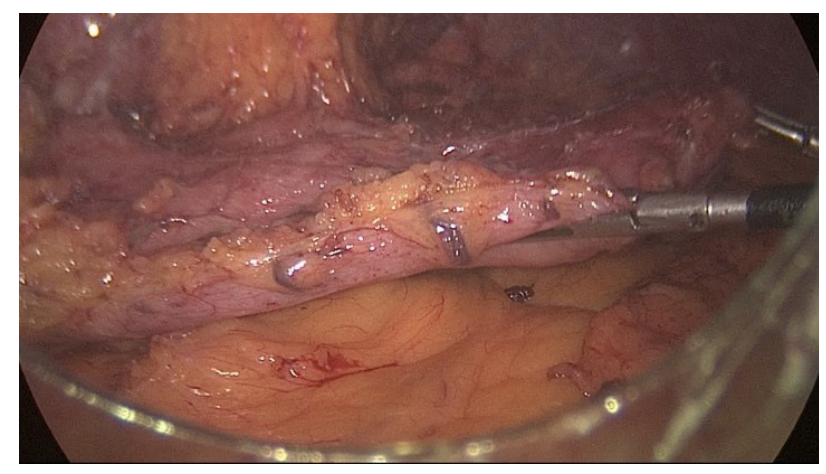

Figura 3. Aspecto final após lise de aderências e confecção do tudo gástrico.

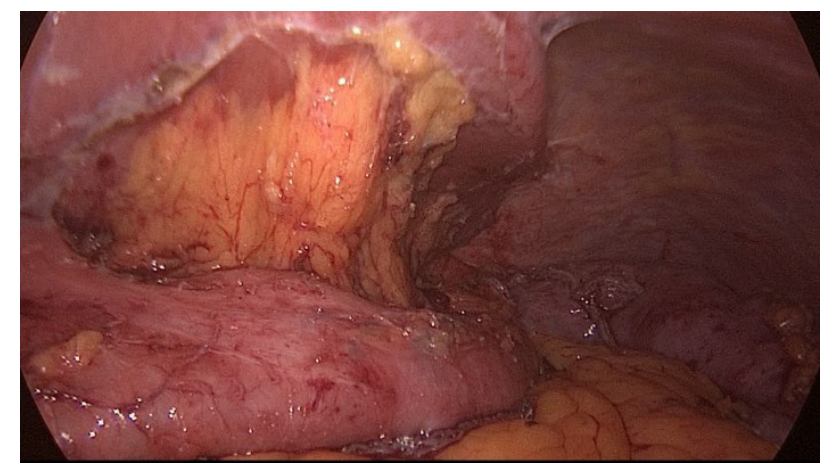

\section{DISCUSSÃO}

A obesidade já configura na atualidade importante problema de saúde pública. Pode ser definida como o excesso de gordura e diagnosticada com IMC acima de 30. ${ }^{5}$ Vários trabalhos mostram a relação com aumento do risco de doenças cardiovasculares, síndromes metabólicas câncer e depressão. ${ }^{6-8}$

A epidemia de obesidade é seguida pelo aumento dramático no número de casos de DHGNA. ${ }^{6}$ A esteatose hepática é definida como o acumulo de lipídeos no citoplasma dos hepatócitos. A esteatose não alcoólica pode ser classificada em 4 tipos: Tipos 1 e 2 que evidenciam infiltrado inflamatório e evolução benigna e tipos 3 e 4 (esteato-hepatite não alcoólica - NASH) que além do infiltrado inflamatório, apresenta alterações degenerativas e fibróticas que podem evoluir para cirrose hepática e carcinoma hepatocelular., ${ }^{7,10}$

Atualmente o NASH é responsável pela maioria dos transplantes de fígado não relacionados a hepatite em diversos países do mundo, o que nos alerta sobre a necessidade de intervenção de sua causa primária, a obesidade. ${ }^{11,12}$ Nos pacientes já submetidos ao transplante hepático, a manutenção de obesidade expõe o paciente ao risco de perda do enxerto. ${ }^{11-13}$

A cirurgia bariátrica é o tratamento de escolha para a obesidade mórbida, sendo proposta para pacientes transplantados e/ ou candidatos, apresentando resultados positivos, com efetividade semelhante à da população geral, podendo ser feita antes, durante ou após o transplante. A técnica mais utilizada é a gastrectomia vertical - sleeve, devido a maior facilidade de execução da técnica, além de não alterar acesso endoscópico ao trato biliar; ou afetar absorção de medicamentos imunossupressores..$^{3,4}$

Em recente metanálise publicada em 2018 na American Society for Bariatric Surgery, foram avaliados um total de 21 estudos, nos quais os pacientes foram submetidos a cirurgia bariátrica para o tratamento de esteatose hepática associada a obesidade. ${ }^{4,13,14}$ Foi observado, assim como em nosso relato de caso, melhora histológica e bioquímica da DHGNA.

Fakhry et al, incluiu 2374 pacientes e teve como resultado: melhora da esteatose em $88 \%$ dos pacientes, a esteato-hepatite melhorou em $59 \%$ e a fibrose melhorou ou foi resolvida em $30 \%$ dos pacientes. O aspartato aminotransferase (TGO) melhorou em $32 \%$ dos pacientes e a alanina aminotransferase (TGP) melhorou em $62 \%$ dos pacientes. ${ }^{4}$ Como podemos observar em nosso estudo e na revisão realizada para este estudo, as evidências atuais sugerem fortemente a cirurgia bariátrica como tratamento da DHGNA.

Em nossa experiência, observamos que a cirurgia bariátrica (Técnica de Sleeve), é segura e eficaz no tratamento da obesidade em pacientes pós-transplante hepático, promovendo excelentes resultados na perda de peso e no tratamento da esteatose hepática severa, podendo ser utilizada como padrão nos casos de DHGNA e risco de perda do enxerto. 


\section{REFERÊNCIAS}

1. Ferreira CT, Vieira SM, Silveira R. Transplante hepático. J Pediatr (Rio J). 2000;76 Supl. 1:S198-208.

2. Starzl TE, Marchioro TL, Von Kaulla K. Homotransplantation of the liver in humans. Surg Gynecol Obstet. 1963;117:659-76.

3. Nassif GB, Salloum C, Paolino L, Azoulay D, Lazzati A. Laparoscopic sleeve gastrectomy after orthotopic liver transplantation, video reported. Obes Surg. 2019;29(4):1436-8.

4. Fakhry TK, Mhaskar R, Schwitalla T, Muradova E, Gonzalvo JP, Murr MM. Bariatric surgery improves nonalcoholic fatty liver disease: a contemporary systematic review and meta-analysis. Surg Obes Relat Dis. 2019;15(3):502-11.

5. Pajecki D, Cesconetto DM, Macacari R, Joaquim H, Andraus W, Cleva R, et al. Cirurgia bariátrica (gastrectomia vertical) após transplante hepático: relato de caso. Arq Bras Cir Dig. 2014;27(3):803.

6. Koebnick C, Getahun D, Reynolds K, Coleman KJ, Porter AH, Lawrence JM, et al. Trends in non-alcoholic fatty liver diseaserelated hospitalizations in US children, adolescents, and young adults. J Pediatr Gastroenterol Nutr. 2009;48(5):597-603.

7. Estes C, Razavi H, Loomba R, Younossi Z, Sanyal AJ. Modeling the epidemic of nonalcoholic fatty liver disease demonstrates an exponential increase in burden of disease. Hepatology. 2018;67(1):123-33.
8. Caldwell S, Argo C. The natural history of non-alcoholic fatty liver disease. Dig Dis 2010;28(1):162-8.

9. Siddiqui MS, Charlton M. Liver transplantation for alcoholic and nonalcoholic fatty liver disease: pretransplant selection and posttransplant management. Gastroenterology. 2016;150(8):1849-62.

10. Shalhub S, Parsee A, Gallagher SF, Haines KL, Willkomm C, Brantley SG, et al. The importance of routine liver biopsy in diagnosing nonalcoholic steatohepatitis in bariatric patients. Obes Surg. 2004;14(1):54-9.

11. Taitano AA, Markow M, Finan JE, Wheeler DE, Gonzalvo JP, Murr MM. Bariatric surgery improves histological features of nonalcoholic fatty liver disease and liver fibrosis. J Gastrointest Surg. 2015;19(3):429-36.

12. Bower G, Toma T, Harling L, Jiao LR, Efthimiou E, Darzi A, et al. Bariatric surgery and non-alcoholic fatty liver disease: a systematic review of liver biochemistry and histology. Obes Surg. 2015;25(12):2280-9.

13. Moher D, Liberati A, Tetzlaff J, Altman DG. Preferred reporting items for systematic reviews and meta-analyses: the PRISMA statement. J Clin Epidemiol. 2009;62(10):1006-12.

14. Higgins J. Cochrane handbook for systematic reviews of interventions: the Cochrane Collaboration. Hoboken: Wiley; 2011.

\section{Como citar:}

Silva MM, Soares CE, Pinto JO, Sales LA, Pinheiro FA, Garcia JH. Gastrectomia vertical (Sleeve) em paciente pós-transplante hepático. Rev Med UFC. 2021;61(1):1-4. 